\title{
Template Matching, Alignment and Averaging of Sub-Tomograms in Electron Cryo-Tomography Reconstructions using Jsubtomo
}

\author{
J.T. Huiskonen ${ }^{1}$ \\ ${ }^{1}$ Oxford Particle Imaging Centre, Division of Structural Biology, Wellcome Trust Centre of Human \\ Genetics, University of Oxford, Oxford, United Kingdom
}

Electron cryo-tomography has emerged as a widely used method for imaging different biological specimens and elucidating their three-dimensional structures. Suitable specimens range from macromolecular complexes, filamentous structures, coated vesicles and pleomorphic membrane viruses to procaryotic cells and thin areas eukaryotic cells. After sample preparation for electron cryo-microscopy, a tomographic tilt series is collected from the sample area of interest, from which a three-dimensional density map (a tomogram) can be calculated. Two complications limit the usefulness of these data: first, low signal-to-noise ratio, inherent to all electron cryo-microscopy data, and second, the missing wedge problem, inherent to all tomographic reconstructions with limited tilting range. However, if the tomogram contains several identical structures (particles), such as macromolecular complexes, these complications can be overcome by averaging several sub-tomograms, each containing a single particle.

Here, a software package named Jsubtomo (www.opic.ox.ac.uk/jsubtomo) for sub-tomogram alignment and averaging is presented. The main goals were to provide a flexible and robust package for users and a documented source code for developers. Jsubtomo has been utilized in the sub-tomogram averaging of several cellular and viral structures [1-6]. The package consists of three main programs: jsubtomo for template matching to detect particles in tomograms, selection of particles and refinement of their locations and orientations, jave for averaging of particles extracted from tomograms, and jviews for incorporating a priori knowledge of locations and orientations of the structures of interest to restrict the refinement in local regions in the tomogram (Figure 1). Furthermore, Python scripts have been implemented for iterative gold-standard refinement.

Different types of simulations were run to test the correctness and robustness of the alignment and averaging algorithms at different amounts of Gaussian noise. In addition to simulations, the software was used to average membrane glycoprotein complexes from experimental tomograms a phlebovirus. This example illustrates the main strength of the package: automatic detection of particles by incorporating a priori knowledge on the particle locations and orientations (Figure 2). Finally, strategies for alignment and averaging of sub-volumes from different types of data are discussed.

References

[1] Huiskonen JT et al. J Virol 84 (2010), p. 4889-97.

[2] Liljeroos L et al. P Natl Acad Sci Usa 108 (2011), p.18085-90.

[3] Karotki L et al. J Cell Biol 195 (2011), p. 889-902.

[4] Pietilä MK et al. J Virol 86 (2012), p. 5067-79.

[5] Bowden TA et al. PLoS Pathog 9 (2013), p. e1003374.

[6] Maurer UE et al. Structure 21 (2013), p. 1396-405. 


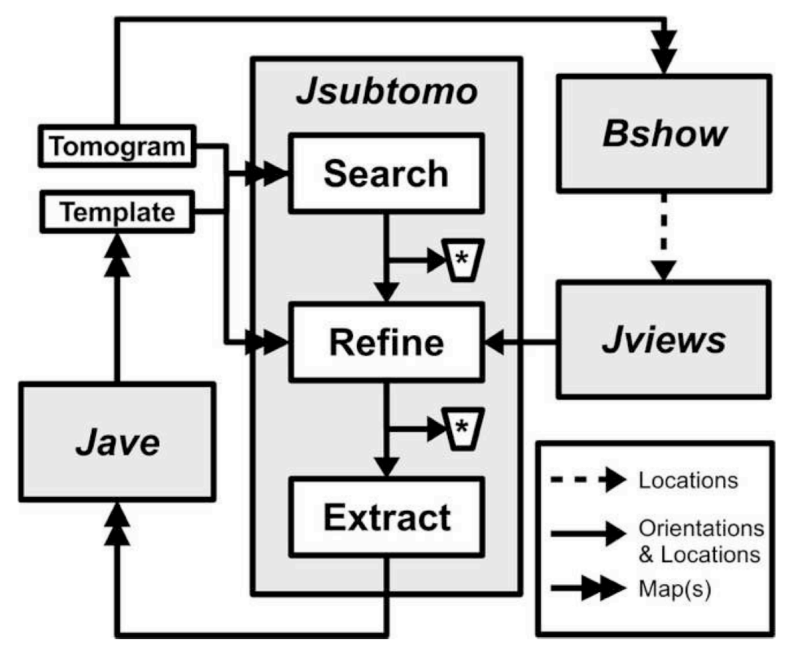

Figure 1. Work-flow of the Jsubtomo package. The steps where false positive particles can be discarded using either a particle-to-particle distance based filter or a cross-correlation coefficient based filter are denoted by an asterisk. Bshow is a visualization tool from the Bsoft package (bsoft.ws).
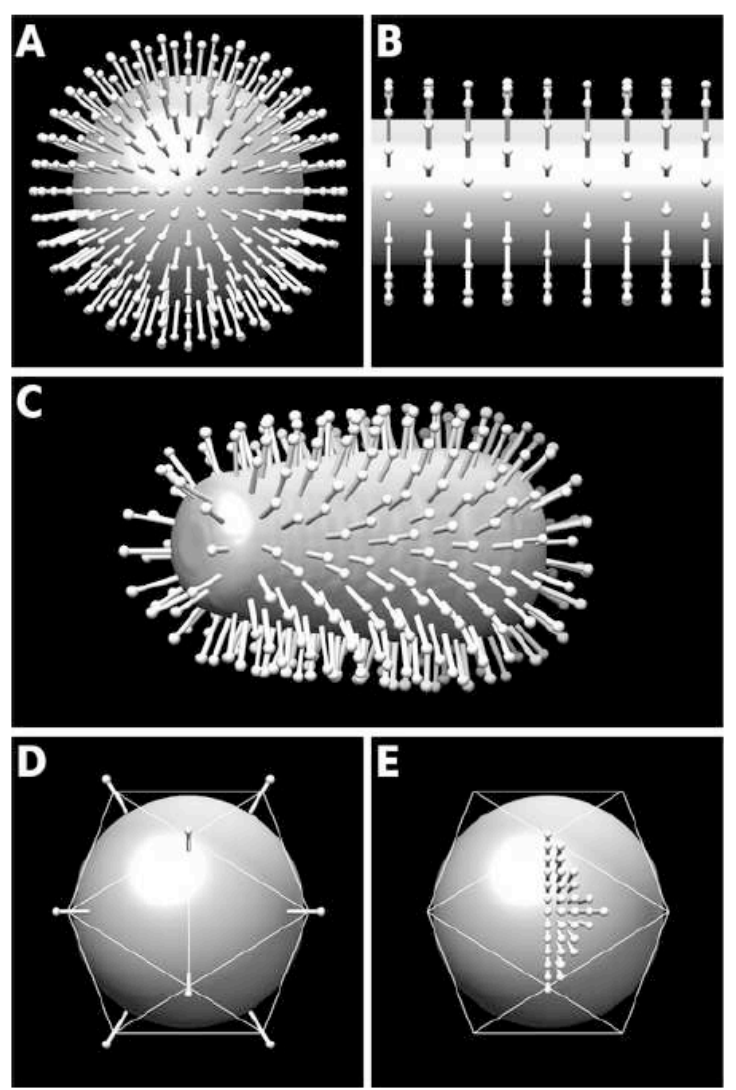

Figure 2. Processing of surface-bound particles. (A-E) Examples of initial particle view vectors ('seeds') reflecting possible surface geometries. Seeds covering a spherical surface $(A)$, a tubular surface $(B)$ and a bullet-shaped surface $(C)$ are shown in addition to seeds located at icosahedral vertexes $(D)$ and seeds covering an icosahedral asymmetric unit $(E)$. 